\title{
Commentary: 10, 7, 17, and 9
}

\author{
Michael Mack, MD
}

From the Cardiovascular Service Line Baylor Scott \& White Health, Dallas, Tex.

Disclosures: Author is uncompensated co-principal investigator of research trials for Abbott, Edwards Lifesciences, and Medtronic.

Received for publication Nov 18, 2018; accepted for publication Nov 19, 2018; available ahead of print Jan 14, 2019.

Address for reprints: Michael Mack, MD, 1100 Allied Dr, Plano, TX 75093 (E-mail: Michael.mack@bswhealth. org).

J Thorac Cardiovasc Surg 2019;157:2198-9

$0022-5223 / \$ 36.00$

Copyright (C) 2018 by The American Association for Thoracic Surgery

https://doi.org/10.1016/j.jtcvs.2018.11.064

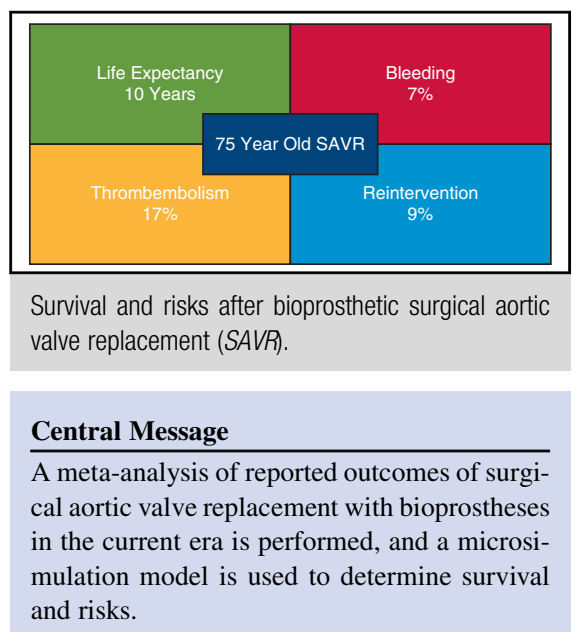

See Article page 2189.
There are multiple series in the literature reporting the longterm outcomes of patients undergoing surgical aortic valve replacement (SAVR) with tissue valves. Most of these series are of limited sample size and are now relatively dated. In this issue of the Journal, Huygens and colleagues ${ }^{1}$ from Rotterdam perform a systematic review and meta-analysis of reported outcomes in the current era after SAVR with bioprostheses in the elderly patient population, defined as older than 65 years. Huygens and colleagues ${ }^{1}$ then use a microsimulation model parametrized with the pooled outcomes of the meta-analysis to extrapolate the results to age-specific life expectancy and lifetime risk of valverelated morbidity.

Huygens and colleagues ${ }^{1}$ review a total of 42 studies reporting 34 patient cohorts including 12,000 patients, with more than 55,000 patient-years of follow-up. Their simulation model shows that a 75-year-old patient after tissue SAVR has an estimated life expectancy of 9.8 years, closely approximating that of the general population of 10.2 years. During that period, the lifetime risk of bleeding is $7 \%$, that of thromboembolism is $17 \%$, and that of reintervention is $9 \%$. For an 85-year-old patient with a life expectancy in the general population of 5.6 years, the estimated life expectancy after SAVR is 5.0, years with lifetime risks of $4.5 \%$ for bleeding, $10.2 \%$ for thromboembolism, and $4.3 \%$ for reintervention.

This study serves a number of important purposes. First, it updates the previous surgical literature regarding the outcomes and performance of bioprosthetic SAVR. Second, the simulation model of an 85-year-old patient serves as a current benchmark for transcatheter aortic valve replacement, which is rapidly replacing surgical AVR in this age population. Third, with the age creep of surgical bioprostheses into progressively younger patients in anticipation of a subsequent transcatheter valve-in-valve procedure, it begins to give us some insight into the current outcomes of SAVR in patients in their 70s. Of course, structural valve deterioration (SVD) is age dependent, and the results presented here cannot be extrapolated to even younger patient cohorts. This analysis, however, does serve as a foundation for subsequent studies to assess outcomes in younger patients.

One of the problems with all outcomes studies that analyze freedom from SVD is that of survivorship bias. One never knows the true freedom from SVD, because it is not known whether nonsurvivors died because of SVD. In addition, one never knows whether patients who actually had SVD did not undergo reintervention either because they were too elderly or ill or elected not to undergo a reoperation. Through the use of their microsimulation model with simulated cohorts and a probabilistic sensitivity analysis, Huygens and colleagues ${ }^{1}$ correct statistically as accurately as possible for the competing risk of death.

The current low-risk trials of aortic stenosis comparing surgical and transcatheter aortic valve replacement with bioprostheses will include follow-up of all of the approximately 2000 patients enrolled in the trials prospectively out to 10 years. These randomized trials will analyze the comparative effectiveness of transcatheter versus surgical bioprosthetic performance in terms of survival and the risks of bleeding, thromboembolism, and SVD. They will also give us validation regarding whether published retrospective analyses, including those with sophisticated analytic methodology, such as this study, are accurate for future observational studies. Until the results of those studies become available periodically during the next 10 years, the best information we have for patient counseling is the information published here. In a typical 75-year-old patient undergoing SAVR, one can expect a life expectancy of 
10 years, a risk of bleeding of $7 \%$, a risk of thromboembolism of $17 \%$ and a risk of need for reintervention for SVD of $9 \%$. The easy way to remember outcome expectations for patient shared decision making is thus $10,7,17$, and 9.

\section{Reference}

1. Huygens SA, Etnel JRG, Hanif M, Bekkers JA, Bogers AJJC, Rutten-van Mölken MPMH, et al. Bioprosthetic aortic valve replacement in elderly patients: meta-analysis and microsimulation. J Thorac Cardiovasc Surg. 2019;157: 2189-97.e14. 